\title{
PROPAGATION AND REFLECTION OF ONE-DIMENSIONAL WAVES IN FERROELECTRIC CERAMICS
}

\author{
BY \\ M. F. MCCARTHY (National University of Ireland, Galway, Ireland) \\ AND
}

T. B. MOODIE, T. S. ÖNCÜ, R. P. SAWATZKY (University of Alberta, Edmonton, Alberta, Canada)

\begin{abstract}
Employing a linearized version of the general constitutive equations for a homogeneous deformable dielectric material body with memory, we study problems of wave propagation and reflection. We formulate these problems so as to correspond to certain well-defined experiments appearing in the literature and involving the application of time-dependent mechanical loads or prescribed voltage differences. Employing asymptotic procedures based upon geometrical optics we are able to solve problems of reflection hitherto unsolved, produce numerical results in an efficient manner, and elicit some important implications of the theory that appear not to have been noted before.
\end{abstract}

1. Introduction. When a polarized specimen of a ferroelectric material is subjected to a mechanical load, electrical responses result, and the application of an external field across it produces mechanical responses; see [1-3]. In addition, a ferroelectric material also exhibits time-dependent responses both mechanically and electrically. This arises because the number of aligned dipoles may be altered by the passage of a mechanical disturbance or the application of an electric field leading to changes in the material properties [4]. Moreover, the material exhibits mechanical dissipation owing to the presence of material defects.

In this paper we examine the detailed behaviour of one-dimensional waves in finite deformable dielectric materials with memory. Our field equations are the linearized version of those developed previously by Chen et al. [5] and employed more recently in this linearized form by Amos and Chen [4, 6]. In the full nonlinear version of the equations studied in [5] the authors extracted much of their information concerning the material's behaviour by means of the theory of acceleration waves. This zero amplitude exact nonlinear theory provides information about the wave only at its leading edge and gives no information concerning the evolution of a pulse. Indeed, to obtain reliable numerical information on the evolution of an arbitrary pulse governed by the full equations of [5] is a very difficult and perhaps intractable problem. 
However, this is not to say that detailed information concerning material response and wave modulation gleaned in some limited regime is not of extreme value in the study of these rather complex materials; for it is.

The first attempts were made by Amos and Chen $[4,6]$ employing the linearized version of the equations [5] and the Laplace transform together with a numerical inversion scheme. There is a shortcoming with this approach, that is, there is no clear description of the reflection process. Moreover, in their treatment of the pulse poling or depoling experiments they employed an assumed electric displacement history which is qualitatively similar to the experimental data. Although employing an assumed electric displacement history was not a crucial ingredient of their method and could have been avoided, it offered some convenience in their numerical calculations.

Here we adopt a method which we feel is more natural to the setting of wave propagation, allowing us a clear description of the reflection process and avoiding the use of an assumed form for the electric displacement history. The displacement history will be obtained from the solution of the initial-boundary value problem. The method adopted for the analysis of the problems considered here is based upon previous results obtained for both thermoviscoelastic [7] and thermoelastic [8] media. This is natural for, as indicated by Chen et al. [5], the problems considered here are generalizations of problems involving deformable materials with memory of which the materials employed in [7] and [8] are examples. By these methods we are able to solve a number of important hitherto unsolved problems involving propagation and reflection, obtain detailed numerical results for the evolution of arbitrary pulses, calculate explicit arrival time formulas for the wave-fronts, and exhibit expressions for the magnitudes of all discontinuities including those for derivatives of all orders.

2. Formulation. We consider a ferroelectric ceramic disc of thickness $L$ that is polarized in the axial direction by attaching electrodes to its two planar surfaces. We choose our frame of reference in such a way that $0<x<L$, where $x$ denotes the axial coordinate of the material point of the disc.

In the absence of body forces and electric charges, the linear field equations for the above-described disc are the law of balance of linear momentum

$$
\rho \frac{\partial^{2} u}{\partial t^{2}}=\frac{\partial T}{\partial x}
$$

and Gauss' law

$$
\frac{\partial D}{\partial x}=0
$$

where $\rho$ is the constant mass density, $u(x, t)$ the mechanical displacement, $T(x, t)$ the stress, $D(t)$ the departure of the electric displacement from an initial electric displacement $D_{r}$ that characterizes the state of residual polarization of the disc, and $t$ the time. That $D$ depends on $t$ alone is a consequence of (2.2). Further, the voltage difference across the disc denoted by $\Phi(t)$ is given by

$$
\Phi=\int_{0}^{L} E(x, t) d x
$$

where $E(x, t)$ is the electric field. 
The linear electromechanical response of the disc is described by the constitutive relations of the stress $T$ and the electric field $E$ which take the forms [5]

$$
\begin{aligned}
T= & \mu(0) S(x, t)+\int_{0}^{\infty} \mu^{\prime}(s) S(x, t-s) d s \\
& +\sigma(0) D(t)+\int_{0}^{\infty} \sigma^{\prime}(s) D(t-s) d s, \\
E= & \omega(0) S(x, t)+\int_{0}^{\infty} \omega^{\prime}(s) S(x, t-s) d s \\
& +\xi(0) D(t)+\int_{0}^{\infty} \xi^{\prime}(s) D(t-s) d s,
\end{aligned}
$$

where $S(x, t)$ is the strain defined by

$$
S=\frac{\partial u}{\partial x}
$$

and $\mu, \sigma$, and $\omega$ may either be relaxation or creep functions whereas $\xi$ is a relaxation function.

In general, the designation of the functions $\mu, \sigma$, and $\omega$ as relaxation or creep functions is related to the decrease or increase of aligned dipoles in the material. This correlation for $\mu$ is not regarded as strong so that $\mu$ may be more correctly designated a relaxation function as its properties appear more strictly dominated by mechanical dissipative effects [9].

Combining the momentum balance equation (2.1) with the constitutive relation for stress (2.4) we obtain the usual wave equation

$$
\rho \frac{\partial^{2} u}{\partial t^{2}}=\mu(0) \frac{\partial^{2} u}{\partial x^{2}}+\int_{0}^{\infty} \mu^{\prime}(s) \frac{\partial^{2} u}{\partial x^{2}}(x, t-s) d s,
$$

for a material with mechanical dissipation. It should be noted that one should not conclude from this equation that the mechanical response of the material is independent of electrical effects. In most physical situations this is not the case as is evident from the constitutive equations (2.4) and (2.5).

In this paper we consider two initial-boundary value problems. For each problem, the material is assumed to be undeformed and at rest prior to $t=0$. Therefore, in both problems the initial conditions for $u(x, t)$ are

$$
u(x, t)=\frac{\partial}{\partial t} u(x, t)=0, \quad(x, t) \in(0, L) \times(-\infty, 0] .
$$

The first (class of) problem(s) to be considered corresponds to a finite resistance experiment where the electrodes attached to the planar surfaces of the disc are connected with a resistive external circuit of resistance $R$. In this case the relevant circuit equation is

$$
-\Phi=i R,
$$

where $i(t)$ is the electric current determined by

$$
i=A \frac{d D}{d t}
$$


and $A$ is the area of each electrode. It then follows from (2.3), (2.5), (2.9), and (2.10) that

$$
\begin{aligned}
& A R \frac{d D}{d t}+L \xi(0) D+L \int_{0}^{\infty} \xi^{\prime}(s) D(t-s) d s \\
& \quad=\omega(0)[u(0, t)-u(L, t)]+\int_{0}^{\infty} \omega^{\prime}(s)[u(0, t-s)-u(L, t-s)] d s .
\end{aligned}
$$

We now assume that transients are excited in the disc as a consequence of disturbances applied at the boundary $x=0$. Thus, either the strain, the stress, or the material velocity is prescribed at $x=0$, that is,

$$
\text { (i) } S(0, t)=S^{*}(t) \text {, }
$$

or

$$
\text { (ii) } T(0, t)=T^{*}(t) \text {, }
$$

or

$$
\text { (iii) } \frac{\partial u}{\partial t}(0, t)=v^{*}(t) \text {. }
$$

The boundary $x=L$ is either rigidly fixed or is stress free, that is,

$$
\text { (a) } u(L, t)=0 \text {. }
$$

or

$$
\text { (b) } T(L, t)=0 \text {. }
$$

Therefore, in this problem Eqs. (2.7) and (2.11) are to be satisfied for every relevant combination of the above boundary conditions and to these must be added an initial condition for $D(t)$. We set

$$
D(0)=0 .
$$

This problem will be called Problem 1.

The second problem is a pulse poling or depoling experiment in which the two planar surfaces of the disc are stress free and transients are excited by introducing a prescribed voltage difference across these surfaces, that is,

$$
\begin{gathered}
T(0, t)=0, \quad T(L, t)=0, \\
\Phi(t)=\Phi^{*}(t) .
\end{gathered}
$$

The basic governing equations for this problem are (2.7) and (2.3). This problem will be referred to as Problem 2.

We shall assume that the functions $\mu(s), \sigma(s), \omega(s)$, and $\xi(s)$ have well-defined 
Taylor series expansions about $t=0$ given by

$$
\begin{array}{rlrl}
\mu(s) & =\sum_{i=0}^{\infty} \mu_{i}^{0} \frac{s^{i}}{i !} ; & \mu_{i}^{0}=\left.\frac{d^{i} \mu}{d s^{i}}\right|_{s=0}, \mu_{0}^{0}>0, \mu_{1}^{0}<0, \\
\sigma(s)=\sum_{i=0}^{\infty} \sigma_{i}^{0} \frac{s^{i}}{i !} ; & \sigma_{i}^{0}=\left.\frac{d^{i} \sigma}{d s^{i}}\right|_{s=0}, \\
\omega(s)=\sum_{i=0}^{\infty} \omega_{i}^{0} \frac{s^{i}}{i !} ; & \omega_{i}^{0}=\left.\frac{d^{i} \omega}{d s^{i}}\right|_{s=0}, \\
\xi(s)=\sum_{i=0}^{\infty} \xi_{i}^{0} \frac{s^{i}}{i !} ; & \xi_{i}^{0}=\left.\frac{d^{i} \xi}{d s^{i}}\right|_{s=0}, \xi_{0}^{0}>0, \xi_{1}^{0}<0 .
\end{array}
$$

It follows from the above conditions imposed on $\mu_{i}^{0}$ and $\xi_{i}^{0}$ that $\mu$ and $\xi$ are relaxation functions while $\sigma$ and $\omega$ may be relaxation or creep functions depending on the values of $\sigma_{0}^{0}$ and $\sigma_{1}^{0}$ and of $\omega_{0}^{0}$ and $\omega_{1}^{0}$.

3. Geometrical optics solution. As the method employed here has been employed previously $[7,8]$ in a restricted form, we shall omit certain of those details from the calculations that have been elaborated upon elsewhere while providing them in those instances that are novel.

The general form of the solutions is

$$
\begin{aligned}
u(x, t) & =\sum_{n=1}^{\infty} u^{(2 n-1)}(x, t)+\sum_{n=1}^{\infty} u^{(2 n)}(x, t), \\
D(t) & =\sum_{n=1}^{\infty} D^{(2 n-1)}(t)+\sum_{n=1}^{\infty} D^{(2 n)}(t) .
\end{aligned}
$$

The components $u^{(2 n-1)}(x, t)$ of the mechanical displacement arise as a result of waves emanating from the boundary $x=0$ while the components $u^{(2 n)}(x, t)$ arise on account of waves emanating from the boundary $x=L$. Similarly, the components $D^{(2 n-1)}(t)$ and $D^{(2 n)}(t)$ of the electric displacement $D(t)$ arise from changes in the electric displacement produced by waves emanating from the boundaries $x=0$ and $x=L$, respectively.

In order to apply the geometrical optics method to solve our problems we first rewrite $(3.1)$ in the form

$$
u(x, t)=\sum_{m=1}^{\infty} u^{(m)}(x, t),
$$

and express each $u^{(m)}$ in terms of their asymptotic expansions

$$
u^{(m)}(x, t)=\sum_{j=1}^{\infty} u_{j}^{(m)}(x) F_{j}\left(t-\theta_{m}(x)\right), \quad u_{j}^{(m)} \equiv 0, j<1 ; m=1,2, \ldots
$$

where

$$
F_{j}^{\prime}=F_{j-1}, \quad j=1,2, \ldots
$$


Thus all of the $F_{j}, j \geq 1$, can be determined from the waveform $F_{0}$ by successively integrating (3.5). In particular, if $F_{0}=H(t)$, where $H(t)$ is the unit step function, then

$$
F_{j}(t)=t^{j} H(t) / j !
$$

To determine the coefficients $u_{j}^{(m)}(x)$ and the phase functions $\theta_{m}(x)$, we now insert (3.3) together with (3.4) and (3.5) into (3.7), employ the Taylor series expansion (2.20) in the result, integrate by parts $(i+1)$ times, and equate the coefficients of $F_{j-1}\left[t-\theta_{m}(x)\right]$. The resulting relationship is

$$
\begin{gathered}
\left(\theta_{m}^{\prime}\right)^{2} \sum_{i=0}^{j-1} \mu_{i}^{0} u_{j-i}^{(m)}-\theta_{m}^{\prime \prime} \sum_{i=0}^{j-2} \mu_{i}^{0} u_{j-i-1}^{(m)}-2 \theta_{m}^{\prime} \sum_{i=0}^{j-2} \mu_{i}^{0} u_{j-i-1}^{(m)^{\prime}}+\sum_{i=0}^{j-3} \mu_{i}^{0} u_{j-i-2}^{(m)^{\prime \prime}}=\rho u_{j}^{(m)}, \\
j=1,2, \ldots ; m=1,2, \ldots
\end{gathered}
$$

The first of the equations in (3.7) is obtained by setting $j=1$. This equation is

$$
\left\{\left[\theta_{m}^{\prime}(x)\right]^{2}-\rho / \mu_{0}^{0}\right\} u_{1}^{(m)}=0 .
$$

Requiring $u_{1}^{(m)} \neq 0$ with no loss of generality, this equation then gives the eikonal equation

$$
\left[\theta_{m}^{\prime}(x)\right]^{2}=\rho / \mu_{0}^{0} .
$$

The eikonal equation integrates to

$$
\theta_{m}(x)= \begin{cases}\tau_{m}+x / V & \text { if } m=2 n-1, n=1,2, \ldots, \\ \tau_{m}+(L-x) / V & \text { if } m=2 n, n=1,2, \ldots,\end{cases}
$$

where

$$
V=\left(\mu_{0}^{0} / \rho\right)^{1 / 2}
$$

is the acoustic wave speed of the material, and $\tau_{2 n-1}$ and $\tau_{2 n}$ represent the set of times at which the waves $u^{(2 n-1)}$ and $u^{(2 n)}$ leave the boundaries $x=0$ and $x=L$, respectively.

Setting $j=2$ in (3.7) gives the first of the so-called transport equations, namely,

$$
u_{1}^{(2 n-1)^{\prime}}-W u_{1}^{(2 n-1)}=0, \quad u_{1}^{(2 n)^{\prime}}+W u_{1}^{(2 n)}=0,
$$

where

$$
W=\frac{-\mu_{1}^{0}}{2 \mu_{0}^{0} V} .
$$

For $2 n-1, n=1,2, \ldots$, the solution of $(3.12)$ is

$$
u_{1}^{(2 n-1)}(x)=\bar{u}_{1}^{(2 n-1)} e^{-W x} ; \quad \bar{u}_{1}^{(2 n-1)}=u_{1}^{(2 n-1)}(0),
$$

whereas for $2 n, n=1,2, \ldots$, it takes the form

$$
u_{1}^{(2 n)}(x)=\bar{u}_{1}^{(2 n)} e^{-W(L-x)} ; \quad \bar{u}_{1}^{(2 n)}=u_{1}^{(2 n)}(L) .
$$


The higher-order transport equations can be obtained and solved to yield

$$
\begin{aligned}
u_{j}^{(2 n-1)}(x)= & \bar{u}_{j}^{(2 n-1)} e^{-W x}+\frac{V}{2 \mu_{0}^{0}} e^{-W x} \\
& \times \int_{0}^{x} e^{W y} \sum_{i=1}^{j}\left\{\mu_{i-1}^{0} u_{j-i-1}^{(2 n-1)^{\prime \prime}}(y)-\frac{2}{V} \mu_{i}^{0} u_{j-i}^{(2 n-1)^{\prime}}(y)\right. \\
& \left.+\frac{1}{V^{2}} \mu_{i+1}^{0} u_{j-i}^{(2 n-1)}(y)\right\} d y, \quad j \geq 2, \\
u_{j}^{(2 n)}(x)= & \bar{u}^{(2 n)} e^{-W(L-x)}+\frac{V}{2 \mu_{0}^{0}} e^{-W(L-x)} \\
& \times \int_{0}^{L-x} e^{W y} \sum_{i=1}^{j}\left\{\mu_{i-1}^{0} u_{j-i-1}^{(2 n)^{\prime \prime}}(y)-\frac{2}{V} \mu_{i}^{0} u_{j-i}^{(2 n)^{\prime}}(y)\right. \\
& \left.+\frac{1}{V^{2}} \mu_{i+1}^{0} u_{j-i}^{(2 n)}(y)\right\} d y, \quad j \geq 2,
\end{aligned}
$$

where $\bar{u}_{j}^{(2 n-1)}=u_{j}^{(2 n-1)}(0)$ and $\bar{u}_{j}^{(2 n)}=u_{j}^{(2 n)}(L)$. It can be proved by induction that the solutions (3.16) and (3.17) are of the form

$$
\begin{aligned}
u_{j}^{(2 n-1)}(x) & =e^{-W x} \sum_{k=0}^{j-1} u_{k j}^{(2 n-1)} x^{k} / k !, \\
u_{j}^{(2 n)}(x) & =e^{-W(L-x)} \sum_{k=0}^{j-1} u_{k j}^{(2 n)}(L-x)^{k} / k !,
\end{aligned}
$$

respectively. The coefficients $u_{k j}^{(m)}$ in (3.18) and (3.19) are determined by the recurrence relation

$$
u_{k j}^{(m)}= \begin{cases}\frac{V}{2 \mu_{0}^{0}} \sum_{i=0}^{j-k-1}\left[\mu_{i}^{0} E^{2}-\frac{2}{V} \mu_{i+1}^{0} E+\frac{1}{V^{2}} \mu_{i+2}^{0}\right] u_{k-1, j-i-1}^{(m)}, & k=1,2, \ldots, j-1, \\ \bar{u}_{j}^{(m)}, & k=0, j=1,2, \ldots, \\ 0, & k<0 \text { or } k \geq 1,\end{cases}
$$

where $E$ is the difference operator defined as

$$
E u_{k j}^{(m)}=u_{k+1, j}^{(m)}-W u_{k j}^{(m)} .
$$

It follows from the above results that the components $D^{(m)}(t), m=1,2, \ldots$, of the electric displacement $D(t)$ must take the form

$$
D^{(m)}(t)=\sum_{j=0}^{\infty} D_{j}^{(m)} F_{j}\left(t-\tau_{m}\right), \quad D_{j}^{(m)}=0, j<0,
$$


where the $D_{j}^{(m)}$ are constants. Therefore the solutions of our problems are complete once $\bar{u}_{j}^{(m)}, D_{j}^{(m)}, \tau_{m}, m=1,2, \ldots$, and $F_{0}$ are determined from the boundary conditions.

Before considering the initial-boundary value problems formulated in the previous section, it is also necessary to obtain the asymptotic expansions for the strain, stress, and voltage difference. Omitting the details of a straightforward but tedious calculation we find from (2.6), (2.4), and (2.3), respectively, that these quantities admit the following expansion.

The strain.

$$
S(x, t)=\sum_{n=1}^{\infty} S^{(2 n-1)}(x, t)+\sum_{n=1}^{\infty} S^{(2 n)}(x, t),
$$

where

$$
\begin{gathered}
S^{(m)}(x, t)=\sum_{j=0}^{\infty} S_{j}^{(m)}(x) F_{j}\left[t-\theta_{m}(x)\right], \quad m=1,2, \ldots, \\
S^{(2 n-1)}(x)=-e^{-W x} \sum_{k=0}^{j}\left(\frac{1}{V} u_{k, j+1}^{(2 n-1)}+W u_{k j}^{(2 n-1)}-u_{k+1, j}^{(2 n-1)}\right) x^{k} / k !, \\
S^{(2 n)}(x)=e^{-W(L-x)} \sum_{k=0}^{j}\left(\frac{1}{V} u_{k, j+1}^{(2 n)}+W u_{k j}^{(2 n)}-u_{k+1, j}^{(2 n)}\right)(L-x)^{k} / k !,
\end{gathered}
$$

and $\bar{S}_{j}^{(2 n-1)}=S_{j}^{(2 n-1)}(0), \bar{S}_{j}^{(2 n)}=S_{j}^{(2 n)}(L)$.

The stress.

$$
T(x, t)=\sum_{n=1}^{\infty}\left\{T_{S}^{(2 n-1)}(x, t)+T_{D}^{(2 n-1)}(t)\right\}+\sum_{n=1}^{\infty}\left\{T_{S}^{(2 n)}(x, t)+T_{D}^{(2 n)}(t)\right\},
$$

where

$$
\begin{aligned}
T_{S}^{(m)}(x, t) & =\sum_{j=0}^{\infty}\left\{\sum_{i=0}^{j} \mu_{i}^{0} S_{j-i}^{(m)}(x)\right\} F_{j}\left[t-\theta_{m}(x)\right] \\
T_{D}^{(m)}(t) & =\sum_{j=0}^{\infty}\left\{\sum_{i=0}^{j} \sigma_{i}^{0} D_{j-i}^{(m)}\right\} F_{j}\left(t-\tau_{m}\right), \quad m=1,2, \ldots
\end{aligned}
$$

The voltage difference.

$$
\begin{aligned}
\Phi(t)= & \sum_{n=1}^{\infty}\left\{\Phi_{L}^{(2 n-1)}(t)-\Phi_{0}^{(2 n-1)}(t)+\Phi_{D}^{(2 n-1)}(t)\right\} \\
& +\sum_{n=1}^{\infty}\left\{\Phi_{L}^{(2 n)}(t)-\Phi_{0}^{(2 n)}(t)+\Phi_{D}^{(2 n)}(t)\right\}
\end{aligned}
$$


where

$$
\begin{aligned}
& \Phi_{L}^{(2 n-1)}(t)=\sum_{j=1}^{\infty}\left\{\sum_{i=0}^{j-1} \omega_{i}^{0} u_{j-i}^{(2 n-1)}(L)\right\} F_{j}\left[t-\theta_{2 n-1}(L)\right], \\
& \Phi_{0}^{(2 n-1)}(t)=\sum_{j=1}^{\infty}\left\{\sum_{i=0}^{j-1} \omega_{i}^{0} \bar{u}_{j-i}^{(2 n-1)}\right\} F_{j}\left(t-\tau_{2 n-1}\right), \\
& \Phi_{L}^{(2 n)}(t)=\sum_{j=1}^{\infty}\left\{\sum_{i=0}^{j-1} \omega_{i}^{0} \bar{u}_{j-i}^{(2 n)}\right\} F_{j}\left(t-\tau_{2 n}\right), \\
& \Phi_{0}^{(2 n)}(t)=\sum_{j=1}^{\infty}\left\{\sum_{i=0}^{j-1} \omega_{i}^{0} u_{j-i}^{(2 n)}(0)\right\} F_{j}\left[t-\theta_{2 n}(0)\right], \\
& \Phi_{D}^{(m)}(t)=\sum_{j=1}^{\infty}\left\{\sum_{i=0}^{j} L \xi_{i}^{0} D_{j-i}^{(m)}\right\} F_{j}\left(t-\tau_{m}\right), \\
& n=1,2, \ldots ; m=1,2, \ldots
\end{aligned}
$$

The above results complete the determination of basic asymptotic expansions satisfying the field, circuit, and constitutive equations. In the next two sections we will specialize these general asymptotic solutions to Problem 1 and Problem 2. We will investigate the two main cases of Problem 1 separately. In the first case, which we call Case (a), the right-hand boundary is rigidly fixed and transients are generated by mechanical disturbances at the other boundary. It will be seen that at $t=0$ such disturbances excite only one wave, which emanates from the left-hand boundary. This wave is reflected at the other boundary at $t=L / V$ and returns to its original source at $t=2 L / V$. For this problem we will determine the solution for all times prior to the arrival of this reflected wave at its original source, that is, for $0<t<2 L / V$. Problem 1/Case (b) differs from Problem 1/Case (a) in that the right-hand boundary is not rigidly fixed but is stress-free. The physical consequence of this difference is that at $t=0$, the disturbances of Problem 1/Case (b) generate two waves; one at $x=0$ and the other at $x=L$. This is the case also for Problem 2. The voltage difference applied across the two stress-free boundaries generates at $t=0$ two waves leaving the boundaries $x=0$ and $x=L$. Therefore, in both problems, each wave is reflected at the opposite boundary at $t=L / V$ arriving back at their original sources at $t=2 L / V$. For the sake of brevity, we will consider reflections only for Problem 2 noting that reflections in Problem 1/Case (b) can be handled in a similar fashion.

4. Problem 1. In this section we consider all physically meaningful combinations of the boundary conditions $(2.12)$ to $(2.16)$. We assume that the disturbances $S^{*}(t), T^{*}(t)$, and $v^{*}(t)$ of the boundary conditions (2.12), (2.13), and (2.14) can be 
expanded in the series

$$
\begin{array}{rlrl}
S^{*}(t) & =\sum_{j=0}^{\infty} S_{j}^{*} F_{j}(t) ; & S_{j}^{*} \equiv 0, j<0, \\
T^{*}(t) & =\sum_{j=0}^{\infty} T_{j}^{*} F_{j}(t) ; & T_{j}^{*} \equiv 0, j<0, \\
v^{*}(t)=\sum_{j=0}^{\infty} v_{j}^{*} F_{j}(t) ; & v_{j}^{*} \equiv 0, j<0,
\end{array}
$$

where $S_{j}^{*}, T_{j}^{*}$, and $v_{j}^{*}$ are constants.

We shall deal first with the boundary conditions at $x=0$. In a sufficiently small right-neighborhood of $(0,0)$ in the $(x, t)$-plane, the solutions $u(x, t)$ and $D(t)$ are given by

$$
\begin{gathered}
u(x, t)=u^{(1)}(x, t)=\sum_{j=1}^{\infty} u_{j}^{(1)}(x) F_{j}(t-x / V), \\
D(t)=D^{(1)}(t)=\sum_{j=0}^{\infty} D_{j}^{(1)} F_{j}(t)
\end{gathered}
$$

and $\tau_{1}=0$.

(i). The strain satisfies the boundary condition (2.12). From (3.23)-(3.25), (4.1), (4.4), and (4.5) it follows that

$$
-\left[\frac{1}{V} \bar{u}_{j+1}^{(1)}+W \bar{u}_{j}^{(1)}-u_{1, j}\right]=S_{j}^{*}, \quad j=0,1, \ldots .
$$

Therefore, the $\bar{u}_{j}^{(1)}$ are given by the recurrence relation

$$
\bar{u}_{j}^{(1)}= \begin{cases}-V S_{0}^{*}, & j=1, \\ -V\left[S_{j-1}^{*}+W \bar{u}_{j-1}^{(1)}-u_{1, j-1}^{(1)}\right], & j=2,3, \ldots\end{cases}
$$

(ii). The stress obeys the boundary condition (2.13). This requires from (3.26), (3.27), (4.2), (4.4), and (4.5) that

$$
\sum_{i=0}^{j}\left(\mu_{i}^{0} \bar{S}_{j-i}^{(1)}+\sigma_{i}^{0} D_{j-i}^{(1)}\right)=T_{j}^{*}, \quad j=0,1, \ldots,
$$

and

$$
\bar{S}_{j}^{(1)}=-\left[\frac{1}{V} \bar{u}_{j+1}^{(1)}+W \bar{u}_{j}^{(1)}-u_{1, j}^{(1)}\right] .
$$

Consequently, the $\bar{u}_{j}^{(1)}$ are determined as

$$
\bar{u}_{j}^{(1)}= \begin{cases}-V \bar{S}_{0}^{(1)}, & j=1, \\ -V\left[\bar{S}_{j-1}^{(1)}+W \bar{u}_{j-1}^{(1)}-u_{1, j-1}^{(1)}\right], & j=2,3, \ldots,\end{cases}
$$


where the $\bar{S}_{j}^{(1)}$ are calculated from

$$
\bar{S}_{j-1}^{(1)}= \begin{cases}\frac{1}{\mu_{0}^{0}}\left[T_{0}^{*}-\sigma_{0}^{0} D_{0}^{(1)}\right], & j=1, \\ \frac{1}{\mu_{0}^{0}}\left[T_{j-1}^{*}-\sigma_{0}^{0} D_{j-1}^{(1)}-\sum_{i=0}^{j-2}\left(\mu_{i+1}^{0} \bar{S}_{j-2-i}^{(1)}+\sigma_{i+1}^{0} D_{j-2-i}^{(1)}\right)\right], & j=2,3, \ldots .\end{cases}
$$

(iii). In this case the velocity is prescribed at $x=0$ as given by (2.14) and (4.3). Thus it follows from these equations and (4.4) that

$$
\bar{u}_{j}^{(1)}=v_{j-1}^{*}, \quad j=1,2, \ldots .
$$

We now consider a particular experiment, which was studied by Amos and Chen [4]. In this experiment the disc is impacted by a linear elastic material "O" with constant velocity $\bar{v}$. It follows from their equation (2.8) that the material velocity at $x=0$ is given by

$$
v^{*}(t)=\frac{\rho_{0} V_{0}}{\rho_{0} V_{0}+\rho V} \bar{v}+\frac{1}{\rho_{0} V_{0}+\rho V^{2} / V_{\dot{u}}}\left[T^{(1)}(0, t)-\mu_{0}^{0} S^{(1)}(0, t)\right],
$$

where $\rho_{0} V_{0}$ is the acoustic impedance of the material "O", $\rho V^{2}=\mu_{0}^{0}$, and $V_{\dot{u}}$ is the speed of the wave associated with each constant particle velocity. In their computations they set $\rho_{0} V_{0}=\rho V$ and $V_{\dot{u}}=V$. We do likewise, and so for our purposes

$$
v^{*}(t)=\frac{\bar{v}}{2}+\frac{V}{2 \mu_{0}^{0}}\left[T^{(1)}(0, t)-\mu_{0}^{0} S^{(1)}(0, t)\right] .
$$

From (3.23)-(3.27), (4.4), and (4.5) it is evident that

$$
\begin{aligned}
T^{(1)}(0, t)-\mu_{0}^{0} S^{(1)}(0, t) \\
\quad=\sum_{j=0}^{\infty}\left[\sigma_{0}^{0} D_{j}^{(1)}+\sum_{i=0}^{j-1}\left(\mu_{i+1}^{0} \bar{S}_{j-1-i}^{(1)}+\sigma_{i+1}^{0} D_{j-1-i}^{(1)}\right)\right] F_{j}(t) .
\end{aligned}
$$

Therefore, (4.3) and (4.15) show that

$$
v_{j}^{*}= \begin{cases}\frac{\bar{v}}{2}+\frac{V \sigma_{0}^{0}}{2 \mu_{0}^{0}} D_{0}^{(1)}, & j=0, \\ \frac{V}{2 \mu_{0}^{0}}\left[\sigma_{0}^{0} D_{j}^{(1)}+\sum_{i=0}^{j-1}\left(\mu_{i+1}^{0} \bar{S}_{j-1-i}^{(1)}+\sigma_{i+1}^{0} D_{j-1-i}^{(1)}\right)\right], & j=1,2, \ldots,\end{cases}
$$

where

$$
\bar{S}_{j-1}^{(1)}= \begin{cases}-\frac{1}{V} \bar{u}_{1}^{(1)}, & j=1, \\ -\left[\frac{1}{V} \bar{u}_{j}^{(1)}+W \bar{u}_{j-1}^{(1)}-u_{1, j-1}^{(1)}\right], & j=2,3, \ldots,\end{cases}
$$

and the $\bar{u}_{j}^{(1)}$ are given recursively by (4.13). The $D_{j}^{(1)}$ will be determined later. Note that in this experiment $F_{0}(t)=H(t)$. 
This completes the discussion of the boundary conditions at $x=0$. Now, we turn our attention to the boundary conditions at $x=L$. We consider just the first wave emanating from $x=L$. The time at which this wave leaves the boundary depends on which boundary condition is applied.

CASE (a). The boundary $x=L$ is rigidly fixed so that the boundary condition is (2.15). Consequently, no wave is excited at $x=L$ until the wave leaving $x=0$ at $t=0$ reaches the boundary $x=L$. Therefore, the $u(x, t)$ given by (4.4) is valid for the whole disc in the interval $0<t<L / V$.

At $t=L / V$ the first wave arrives at $x=L$ and is reflected. This shows that $\tau_{2}$ of (3.10) is $\tau_{2}=L / V$ so that after reflection the $u(x, t)$ becomes

$$
\begin{aligned}
u(x, t) & =u^{(1)}(x, t)+u^{(2)}(x, t) \\
& =\sum_{j=1}^{\infty} u_{j}^{(1)}(x) F_{j}(t-x / V)+\sum_{j=1}^{\infty} u_{j}^{(2)} F_{j}(t-(2 L-x) / V) .
\end{aligned}
$$

This solution is valid over the whole disc until the reflected wave returns to its original source, that is, until $t=2 L / V$. Since the boundary condition at $x=L$ does not involve $D(t)$, it does not change when the first wave leaves $x=L$ (nor does it change when subsequent waves leave $x=L)$. Subsequently, the solutions $u(x, t)$ and $D(t)$ valid in the interval $0<t<2 L / V$ over the whole disc are given by (4.18) and (4.5), respectively.

It follows from (2.15) and (4.18) that

$$
u^{(1)}(L, t)+u^{(2)}(L, t)=0,
$$

so that

$$
\bar{u}_{j}^{(2)}-u_{j}^{(1)}(L), \quad j=1,2, \ldots .
$$

Therefore, with the use of (3.18), the $\bar{u}_{j}^{(2)}$ are determined as

$$
\bar{u}_{j}^{(2)}=-e^{-W L} \sum_{k=0}^{j-1} u_{k j}^{(1)} L^{k} / k !, \quad j=1,2, \ldots
$$

Lastly, we turn our attention to determining $D(t)$ for Case (a). The use of (4.5), (4.18), and (4.19) in (2.11) gives

$$
R A \sum_{j=0}^{\infty} D_{j+1}^{(1)} F_{j}(t)=\sum_{j=1}^{\infty}\left[\sum_{i=0}^{j-1} \omega_{i}^{0} \bar{u}_{j-i}^{(1)}\right] F_{j}(t)-\sum_{j=1}^{\infty}\left[\sum_{i=0}^{j} L \xi_{i}^{0} D_{j-i}^{(1)}\right] F_{j}(t),
$$

and $D_{0}^{(1)}=0$ from the initial condition $D(0)=0$. This shows that the $D_{j}^{(1)}$ are given by the recurrence relation

$$
D_{j}^{(1)}= \begin{cases}0, & j=0,1, \\ \frac{1}{R A} \sum_{i=0}^{j-2}\left[\omega_{i}^{0} \bar{u}_{j-1-i}^{(1)}-L \xi_{i}^{0} D_{j-1-i}^{(1)}\right], & j=2,3, \ldots,\end{cases}
$$

and the solution of Case (a) for $0<t<2 L / V$ is complete. 
CASE (b). The boundary $x=L$ is stress free so that the boundary condition is (2.16). Since the stress $T(L, t)$ at $x=L$ depends on the electric displacement $D(t)$, and $D(t)$ changes at $t=0$ on account of the wave leaving $x=0$ at $t=0$, $u(L, t)$ must change accordingly at $t=0$. That is, a wave is excited at $x=L$ at $t=0$.

Thus in a sufficiently small left-neighborhood of the point $(L, 0)$ in the $(x, t)$ plane, since $\tau_{2}=0$,

$$
\begin{gathered}
u(x, t)=u^{(2)}(x, t)=\sum_{j=1}^{\infty} u^{(2)}(x) F_{j}(t-(L-x) / V), \\
D(t)=D^{(1)}(t)=\sum_{j=0}^{\infty} D_{j}^{(1)} F_{j}(t) .
\end{gathered}
$$

Note that, as in Case (a), $D(t)$ does not contain a component $D^{(2)}(t)$. This is because $\tau_{1}=\tau_{2}=0$, so it is not necessary to distinguish between the components $D^{(1)}(t)$ and $D^{(2)}(t)$. It is convenient to leave the single component with the name $D^{(1)}(t)$.

With (4.24) and (4.25), from (3.26) and (3.27) the stress $T(x, t)$ at $x=L$ becomes

$$
T(L, t)=\sum_{j=0}^{\infty}\left[\sum_{i=0}^{j} \mu_{i}^{0} \bar{S}_{j-i}^{(2)}+\sigma_{i}^{0} D_{j-i}^{(1)}\right] F_{j}(t),
$$

where

$$
\bar{S}_{j}^{(2)}=\frac{1}{V} \bar{u}_{j+1}^{(2)}+W \bar{u}_{j}^{(2)}-u_{i, j}^{(2)} .
$$

Therefore (2.16) is satisfied if

$$
\sum_{i=0}^{j}\left(\mu_{i}^{0} \bar{S}_{j-i}^{(2)}+\sigma_{i}^{0} D_{j-i}^{(1)}\right)=0, \quad j=0,1,2, \ldots
$$

This provides the following recurrence relation for $\bar{u}_{j}^{(2)}$ :

$$
\bar{u}_{j}^{(2)}= \begin{cases}\frac{1}{V} \bar{S}_{0}^{(2)}, & j=1, \\ \frac{1}{V}\left[\bar{S}_{j-1}^{(2)}-W \bar{u}_{j-1}^{(2)}+u_{1, j-1}^{(1)}\right], & j=2,3, \ldots,\end{cases}
$$

where

$$
\bar{S}_{j-1}^{(2)}= \begin{cases}-\frac{\sigma_{0}^{0}}{\mu_{0}^{0}} D_{0}^{(1)}, & j=1, \\ -\frac{1}{\mu_{0}^{0}}\left[\sigma_{0}^{0} D_{j-1}^{(1)}+\sum_{i=0}^{j-2}\left(\mu_{i+1}^{0} \bar{S}_{j-2-i}^{(2)}+\sigma_{i+1}^{0} D_{j-2-i}^{(1)}\right)\right], & j=2,3, \ldots\end{cases}
$$


To complete the solution for $0<t<L / V$, it remains to determine the components $D_{j}^{(1)}$. In the region of interest the equation (2.11) becomes

$$
R A \sum_{j=0}^{\infty} D_{j+1}^{(1)} F_{j}(t)=\sum_{j=1}^{\infty}\left[\sum_{i=0}^{j-1} \omega_{i}^{0}\left(\bar{u}_{j-i}^{(1)}-\bar{u}_{j-i}^{(2)}\right)\right] F_{j}(t)-\sum_{j=1}^{\infty}\left[\sum_{i=0}^{j} L \xi_{i}^{0} D_{j-i}^{(1)}\right] F_{j}(t),
$$

and $D_{0}^{(1)}=0$ again from the initial condition $D(0)=0$. Therefore the recurrence relation for the $D_{j}^{(1)}$ is

$$
D_{j}^{(1)}= \begin{cases}0, & j=0,1, \\ \frac{1}{R A} \sum_{i=0}^{j-2}\left[\omega_{i}^{0}\left(\bar{u}_{j-1-i}^{(1)}-\bar{u}_{j-1-i}^{(2)}\right)-L \xi_{i}^{0} D_{j-1-i}^{(1)}\right], & j=2,3, \ldots .\end{cases}
$$

This completes our discussion on the solution for Case (b). The solution has been calculated over the interval $0<t<L / V$ and, over the whole disc, the $u(x, t)$ is given by

$$
\begin{aligned}
u(x, t) & =u^{(1)}(x, t)+u^{(2)}(x, t) \\
& =\sum_{j=1}^{\infty} u_{j}^{(1)}(x) F_{j}(t-x / V)+\sum_{j=1}^{\infty} u_{j}^{(1)}(x) F_{j}(t-(L-x) / V),
\end{aligned}
$$

whereas the $D(t)$ is given by (4.25).

Before closing this section we remark that when the external circuit is open, $R=$ $\infty$. From (4.22) and (4.32) it can be seen that, for both Case (a) and Case (b), $D_{j}^{(1)} \rightarrow 0$ as $R \rightarrow \infty$. It then follows from (4.29) and (4.30) that $\bar{u}_{j}^{(2)} \rightarrow 0$ for Case (b). Consequently, the voltage difference across the disc is identical for both cases as these cases become equivalent. In this case the voltage difference occurs solely because of the mechanical strain in the disc. Indeed, it is not necessary to use an asymptotic analysis to come to these conclusions. It follows from the condition $D(0)=0$ and $(2.11)$ that as $R \rightarrow \infty, D(t) \rightarrow 0$. Therefore, when the boundary $x=L$ is stress free, it is necessary that $u(L, t)=0$ and the voltage difference across the disc is

$$
\Phi(t)=\int_{0}^{L} E(x, t) d x=-\left[\omega(0) u(0, t)+\int_{0}^{\infty} \omega^{\prime}(s) u(0, t-s) d s\right] .
$$

5. Problem 2. In this problem transients are excited as a result of the voltage difference $\Phi^{*}(t)$ applied across the two faces of the disc. Suppose that $\Phi^{*}(t)$ can be expanded in the series

$$
\Phi^{*}(t)=\sum_{j=0}^{\infty} \Phi_{j}^{*} F_{j}(t), \quad \Phi_{j}^{*} \equiv 0, j<0,
$$

where $\Phi_{j}^{*}$ are constants.

It follows from the boundary conditions $(2.18)$ that this applied voltage difference excites a wave at $x=0$ and another wave at $x=L$ at time $t=0$. This shows that 
the departure times $\tau_{1}$ and $\tau_{2}$ are zero and the solutions $u(x, t)$ and $D(t)$ valid over the interval $0<t<L / V$ are given by

$$
\begin{gathered}
u(x, t)=u^{(1)}(x, t)+u^{(2)}(x, t) \\
=\sum_{j=1}^{\infty} u_{j}^{(1)}(x) F_{j}(t-x / V)+\sum_{j=1}^{\infty} u_{j}^{(2)}(x) F_{j}(t-(L-x) / V), \\
D(t)=u^{(1)}(t)=\sum_{j=0}^{\infty} D_{j}^{(1)} F_{j}(t) .
\end{gathered}
$$

Employing (5.2) and (5.3) in the expansions (3.26), (3.27), and (3.28), (3.29) we find that the stress and voltage difference take the forms

$$
T(x, t)=T^{(1)}(x, t), \quad \Phi(t)=\Phi^{(1)}(t),
$$

where

$$
\begin{gathered}
T^{(1)}(x, t)=\sum_{j=0}^{\infty}\left\{\sum_{i=0}^{j} \mu_{i}^{0} S_{j-1}^{(1)}(x)\right\} F_{j}(t-x / V) \\
+\sum_{j=0}^{\infty}\left\{\sum_{i=0}^{j} \mu_{i}^{0} S_{j-i}^{(2)}(x)\right\} F_{j}(t-(L-x) / V) \\
+\sum_{j=0}^{\infty}\left\{\sum_{i=0}^{\infty} \sigma_{i}^{0} D_{j-i}^{(1)}\right\} F_{j}(t), \\
\Phi^{(1)}(x, t)=\sum_{j=1}^{\infty}\left\{\sum_{i=0}^{j-1} \omega_{i}^{0}\left(\bar{u}_{j-i}^{(2)}-\bar{u}_{j-i}^{(1)}\right)\right\} F_{j}(t)+\sum_{j=0}^{\infty}\left\{\sum_{i=0}^{j} L \xi_{i}^{0} D_{j-i}^{(1)}\right\} F_{j}(t) .
\end{gathered}
$$

The stress satisfies the boundary condition at $x=0$ if $T^{(1)}(0, t)=0$, which requires that

$$
\sum_{i=0}^{j}\left(\mu_{i} \bar{S}_{j-i}^{(1)}+\sigma_{i}^{0} D_{j-i}^{(1)}\right)=0, \quad j=0,1,2, \ldots
$$

where

$$
\bar{S}_{j}^{(1)}=-\frac{1}{V}\left[\bar{u}_{j+1}^{(1)}+W \bar{u}_{j}^{(1)}-u_{1, j-1}^{(1)}\right] .
$$

Therefore the $\bar{u}_{j}^{(1)}$ are given by the recurrence relation

$$
\bar{u}_{j}^{(1)}= \begin{cases}-V \bar{S}_{0}^{(1)}, & j=1, \\ -V\left[S_{j-1}^{(1)}+W \bar{u}_{j-1}^{(1)}-u_{1, j-1}^{(1)}\right], & j=2,3, \ldots,\end{cases}
$$

where

$$
\bar{S}_{j-1}^{(1)}= \begin{cases}-\frac{\sigma_{0}^{0}}{\mu_{0}^{0}} D_{0}^{(1)}, & j=1, \\ -\frac{1}{\mu_{0}^{0}}\left[\sigma_{0}^{0} D_{j-1}^{(1)}+\sum_{i=0}^{j-2}\left(\mu_{i+1}^{0} \bar{S}_{j-2-i}^{(1)}+\sigma_{i+1}^{0} D_{j-2-i}^{(1)}\right)\right], & j=2,3, \ldots .\end{cases}
$$


Similarly, the boundary condition $T^{(1)}(L, t)=0$ leads to the recurrence relation

$$
\bar{u}_{j}^{(2)}= \begin{cases}V \bar{S}_{0}^{(2)}, & j=1, \\ V\left[\bar{S}_{j-1}^{(2)}-W \bar{u}_{j-1}^{(2)}+u_{1, j-1}^{(2)}\right], & j=2,3, \ldots,\end{cases}
$$

where

$$
\bar{S}_{j-1}^{(2)}= \begin{cases}-\frac{\sigma_{0}^{0}}{\mu_{0}^{0}} D_{0}^{(1)}, & j=1, \\ -\frac{1}{\mu_{0}^{0}}\left[\sigma_{0}^{0} D_{j-1}^{(1)}+\sum_{i=1}^{j-2}\left(\mu_{i+1}^{0} \bar{S}_{j-2-i}^{(2)}+\sigma_{i+1}^{0} D_{j-2-i}^{(1)}\right)\right], & j=2,3, \ldots .\end{cases}
$$

Note that $\bar{S}_{j}^{(2)}=\bar{S}_{j}^{(1)}$ and $\bar{u}_{j}^{(2)}=-\bar{u}_{j}^{(1)}$. Indeed, this result is self-evident from the symmetry of the boundary conditions.

Now, we seek to satisfy the condition (2.19), which demands that

$$
\Phi^{(1)}(t)=\Phi^{*}(t) \text {. }
$$

Consequently, it follows from (5.13), (5.6), and (5.1) that

$$
D_{j}^{(1)}= \begin{cases}\frac{\Phi_{0}^{*}}{L \xi_{0}^{0}}, & j=0, \\ \frac{1}{L \xi_{0}^{0}}\left\{\Phi_{j}^{*}+\sum_{i=0}^{j-1}\left[\omega_{i}^{0}\left(\bar{u}_{j-i}^{(1)}-\bar{u}_{j-i}^{(2)}\right)-L \xi_{i+1}^{0} D_{j-1-i}^{(1)}\right]\right\}, & j=1,2, \ldots\end{cases}
$$

Therefore, the solution valid in the interval $0<t<L / V$ is complete.

At $t=L / V$ the waves $u^{(1)}(x, t)$ and $u^{(2)}(x, t)$ arrive at their destinations and are reflected. The departure times $\tau_{3}$ and $\tau_{4}$ of these reflected waves $u^{(3)}(x, t)$ and $u^{(4)}(x, t)$ are $\tau_{3}=\tau_{4}=L / V$. Thus the solutions $u(x, t)$ and $D(t)$ valid in the interval $0<t<2 L / V$ are

$$
\begin{gathered}
u(x, t)=u^{(1)}(x, t)+u^{(2)}(x, t)+u^{(3)}(x, t)+u^{(4)}(x, t) \\
=\sum_{j=1}^{\infty} u_{j}^{(1)}(x) F_{j}(t-x / V)+\sum_{j=1}^{\infty} u_{j}^{(2)}(x) F_{j}(t-(L-x) / V) \\
+\sum_{j=1}^{\infty} u_{j}^{(3)}(x) F_{j}(t-L(L+x) / V)+\sum_{j=1}^{\infty} u_{j}^{(4)}(x) F_{j}(t-(2 L-x) / V) \\
D(t)=D^{(1)}(t)+D^{(3)}(t) \\
=\sum_{j=0}^{\infty} D_{j}^{(1)} F_{j}(t)+\sum_{j=0}^{\infty} D_{j}^{(3)} F_{j}(t-L / V)
\end{gathered}
$$

while $T(x, t)$ and $\Phi(t)$ become

$$
T(x, t)=T^{(1)}(x, t)+T^{(2)}(x, t), \quad \Phi(t)=\Phi^{(1)}(t)+\Phi^{(2)}(t),
$$


where

$$
\begin{aligned}
T^{(2)}(x, t)= & \sum_{j=0}^{\infty}\left\{\sum_{i=0}^{j} \mu_{i}^{0} S_{j-i}^{(3)}(x)\right\} F_{j}(t-(L+x) / V) \\
& +\sum_{j=0}^{\infty}\left\{\sum_{i=0}^{j} \mu_{i}^{0} S_{j-i}^{(4)}(x)\right\} F_{j}(t-(2 L-x) / V) \\
& +\sum_{j=0}^{\infty}\left\{\sum_{i=0}^{j} \sigma_{i}^{0} D_{j-i}^{(3)}\right\} F_{j}(t-L / V), \\
\Phi^{(2)}(t)= & \sum_{j=1}^{\infty}\left\{\sum_{i=0}^{j-1} \omega_{i}^{0}\left(u_{j-i}^{(1)}(L)-u_{j-i}^{(2)}(0)\right)\right\} F_{j}(t-L / V) \\
+ & \sum_{j=1}^{\infty}\left\{\sum_{i=0}^{j-1} \omega_{i}^{0}\left(\bar{u}_{j-i}^{(4)}-\bar{u}_{j-i}^{(3)}\right)\right\} F_{j}(t-L / V) \\
& +\sum_{j=0}^{\infty}\left\{\sum_{i=0}^{j} L \xi_{i}^{0} D_{j-i}^{(3)}\right\} F_{j}(t-L / V),
\end{aligned}
$$

and $T^{(1)}(x, t)$ and $\Phi^{(1)}(t)$ are defined by (5.5) and (5.6), respectively.

In the interval $L / V<t<2 L / V$ the boundary condition $T(0, t)=0$ becomes

$$
T^{(1)}(0, t)+T^{(2)}(0, t)=0
$$

where

$$
T^{(1)}(0, t)=\sum_{j=0}^{\infty}\left\{\sum_{i=0}^{j} \mu_{i}^{0} S_{j-i}^{(2)}(0)\right\} F_{j}(t-L / V)
$$

and

$$
T^{(2)}(0, t)=\sum_{j=0}^{\infty}\left\{\sum_{i=0}^{j}\left(\mu_{i}^{0} \bar{S}_{j-i}^{(3)}+\sigma_{i}^{0} D_{j-i}^{(3)}\right)\right\} F_{j}(t-L / V) .
$$

The implication of $(5.20),(5.21)$, and (5.22) is the recurrence relation for $\bar{u}_{j}^{(3)}$ given by

$$
\bar{u}_{j}^{(3)}= \begin{cases}-V \bar{S}_{0}^{(3)}, & j=1, \\ -V\left[\bar{S}_{j-1}^{(3)}+W \bar{u}_{j-1}^{(3)}-u_{1, j-1}^{(3)}\right], & j=2,3, \ldots,\end{cases}
$$

where

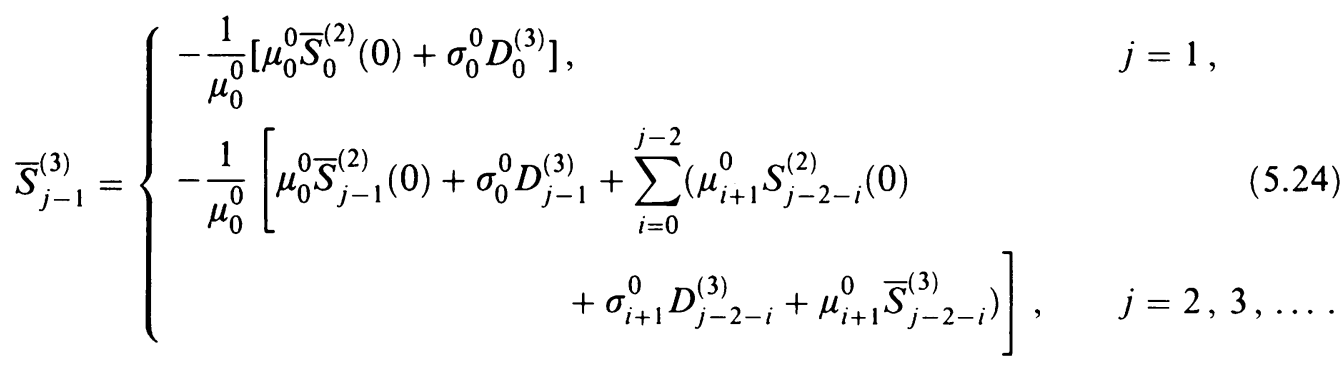


Without duplicating the work, we obtain from the boundary condition $T(L, t)=0$ the following recurrence relation for $\bar{u}_{j}^{(4)}$ :

$$
\bar{u}_{j}^{(4)}= \begin{cases}-V \bar{S}_{0}^{(4)}, & j=1, \\ -V\left[\bar{S}_{j-1}^{(4)}+W \bar{u}_{j-1}^{(4)}-u_{1, j-1}^{(4)}\right], & j=2,3, \ldots,\end{cases}
$$

where

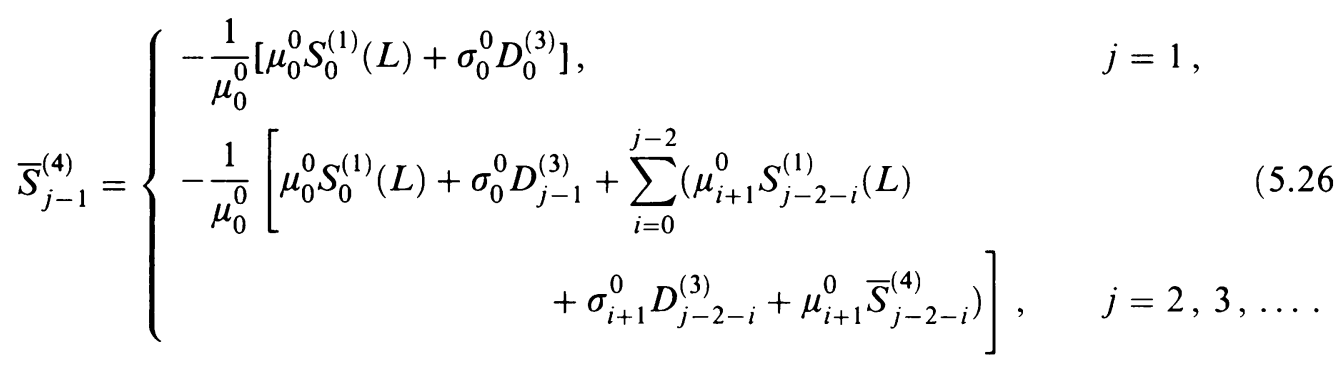

Lastly, we seek to determine the $D_{j}^{(3)}$ from the condition (2.19). The condition (2.19), together with (5.13), (5.17), and (5.19), implies that

$$
\Phi^{(2)}(t)=0 .
$$

Thus the $D_{j}^{(3)}$ obey the recurrence relation

$$
D_{j}^{(3)}=\left\{\begin{array}{rr}
0, & j=0, \\
\frac{1}{L \xi_{0}^{0}} \sum_{i=0}^{j-1}\left[\omega_{i}^{0}\left(\bar{u}_{j-i}^{(3)}+u_{j-i}^{(2)}(0)-\bar{u}_{j-i}^{(4)}-u_{j-i}^{(1)}(L)\right)\right. & \\
\left.-L \xi_{i+1}^{0} D_{j-1-i}^{(3)}\right], & j=1,2, \ldots .
\end{array}\right.
$$

With the above results the solution to Problem 2 over the time interval $0<t<$ $2 L / V$ is complete. The computation of the solution for larger times is straightforward. The subsequent reflections at $x=0$ and $x=L$ can be handled in the same way that the first reflected waves were dealt with. In fact, no further calculations are required. The formulae for the first waves can be modified easily to account for subsequent reflections, as long as the components of the solution that are caused by each reflection are clearly identified.

6. Results. All numerical results have been achieved by means of ten by ten Padé approximants applied to the linear geometrical optics solutions [8]. In all seven figures we employ the explicit representations for the relaxation and/or creep functions given by

$$
\begin{aligned}
\mu(s) & =(1-a) \mu_{0}^{0} e^{-s / \tau_{\mu}}+a \mu_{0}^{0} ; \quad \mu_{0}^{0}>0,0<a<1, \\
\sigma(s) & =(1-b) \sigma_{0}^{0} e^{-s / \tau_{\sigma}}+b \sigma_{0}^{0}, \\
\omega(s) & =(1-c) \omega_{0}^{0} e^{-s / \tau_{\omega}}+c \omega_{0}^{0}, \\
\xi(x) & =(1-d) \xi_{0}^{0} e^{-s / \tau_{\xi}}+d \xi_{0}^{0} ; \quad \xi_{0}^{0}>0,0<d<1,
\end{aligned}
$$


choosing the parameter values to coincide with those selected by Amos and Chen [4] to represent certain experimental configurations. Also, the various physical quantities are taken to have the fixed numerical values specified as follows: $\rho_{0}=7.8 \mathrm{gm} / \mathrm{cm}^{3}$, $\mu_{0}^{0}=158 \mathrm{GPA}, \sigma_{0}^{0}=\omega_{0}^{0}=225 \mathrm{kV} / \mathrm{cm}, \xi_{0}^{0}=10^{5} \mathrm{kV} \mathrm{cm} / C, \tau_{\mu}=0.5 \mu \mathrm{sec}, \tau_{\xi}=$ $0.4 \mu \mathrm{sec}, a=0.9, d=0.75, L=0.25 \mathrm{~cm}, A=1 \mathrm{~cm}^{2}, R=1.0 \Omega$. It should also be noted that although we chose the specific forms in (6.1)-(6.4), our method is not restricted to these forms only but can handle a wide class of relaxation or creep functions.

Figures 1-3 (see pp. 236 and 237) refer to the finite resistance experiment investigated by Amos and Chen [4] and specified here as Problem 1, Case (a)-(iii). Figure 1 shows current plotted versus time and is included principally as a check on our numerical scheme and also provides a check on the scheme employed in [4]. In Figs. 2 and 3 we plot the electric field versus time at three separate stations and for two sets of parameter values. Our results show clearly the utility of the linear geometrical optics approach in that we can capture the reflected waves. This is in contrast to the small time results presented by Amos and Chen [4] in which reflections are not included. The significance of the particular choice of physical parameters employed in these figures has been discussed in detail in Amos and Chen [4].

Figures 4-7 (see pp. 237-239) refer to our Problem 2 which is related to the polingdepoling experiments studied in [4]. In all of these figures the voltage difference is fixed at $0.125 \mathrm{kV}$. In Fig. 4 we show the calculated electric displacement history for various parameter regimes. Apart from the fact that we obtain this quantity as part of our solution it is noteworthy that its behaviour is relatively insensitive to the choice of parameter. This serves to confirm certain experimental observations and to justify a single choice for $D(t)$ as was done in [4]. Our calculated value of $D(t)$ differs from the invoked one employed in [4] and leads to results for the boundary displacement which although qualitatively similar to those in [4] have quantitative differences as displayed in Fig. 5.

In Figs. 6 and 7 we plot the electric field as a function of time at various stations interior to the disc for both the poling and depoling experiments, respectively. These figures clearly demonstrate the effect of the stress free boundary at $x=L$ and the coupling between mechanical and electrical disturbances. Waves are initiated simultaneously both at $x=0$ and $x=L$ at $t=0$. 


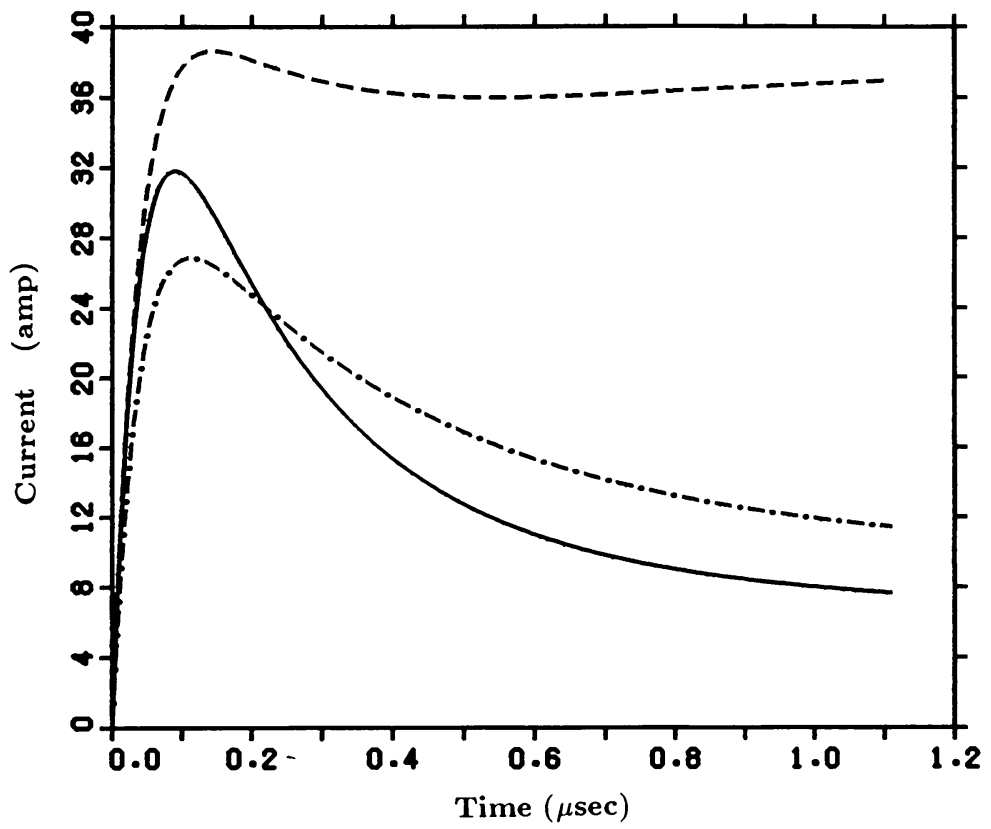

Fig. 1. Current with time for $b=c=0.1, \tau_{\sigma}=\tau_{\omega}=0.2 \mu \mathrm{sec}$, $\bar{v}=0.01 \mathrm{~cm} / \mu \mathrm{sec}(-), b=c=0.6, \tau_{\sigma}=\tau_{\omega}=0.2 \mu \mathrm{sec}, \bar{v}=$ $0.01 \mathrm{~cm} / \mu \mathrm{sec}(--), \quad b=c=0.2, \tau_{\sigma}=\tau_{\omega}=0.3 \mu \mathrm{sec}, \bar{v}=$ $0.0075 \mathrm{~cm} / \mu \mathrm{sec}(-\cdot-)$.

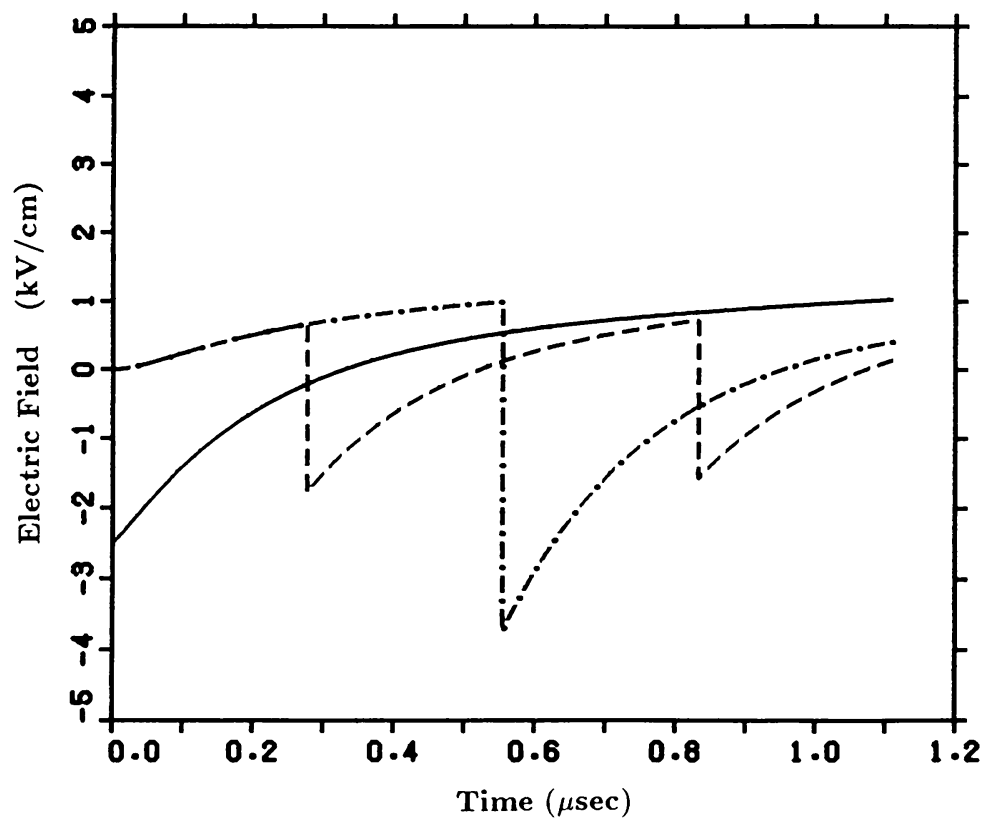

Fig. 2. Electric field with time for $b=c=0.1, \tau_{\sigma}=\tau_{\omega}=0.2 \mu \mathrm{sec}$, $\bar{v}=0.01 \mathrm{~cm} / \mu \mathrm{sec}$ at $x=0(-), x=1 / 2(--)$, and $x=L$ $(-\cdot-)$. 


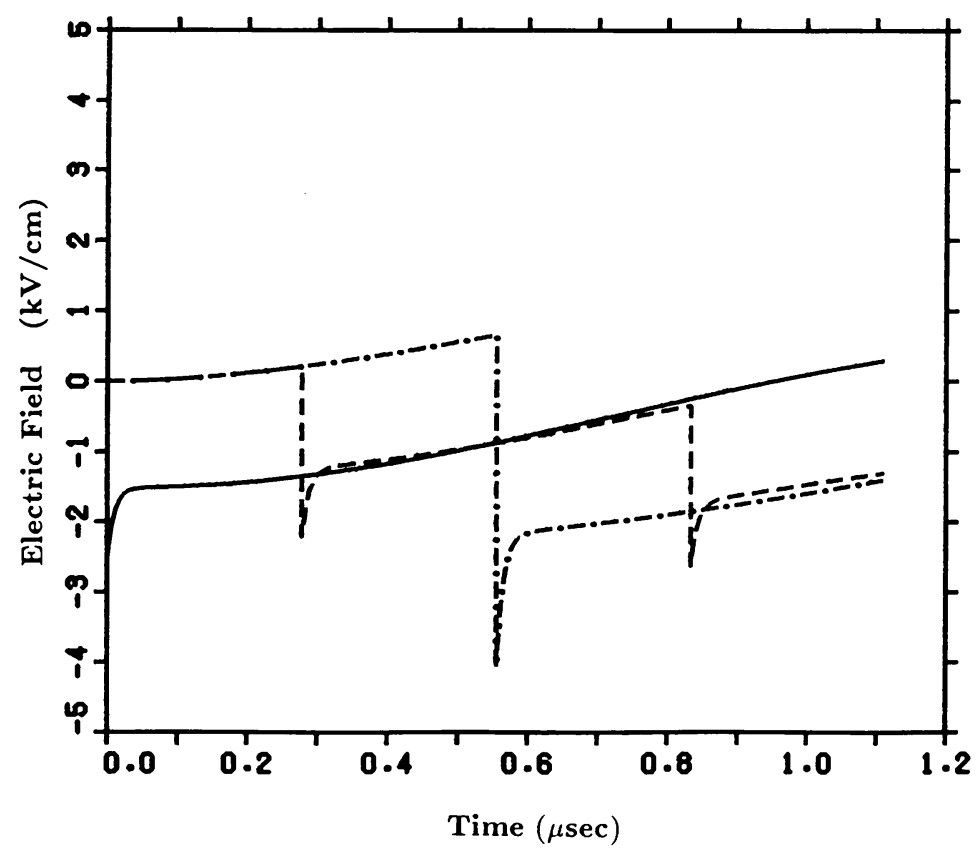

FIG. 3. Electric field with time for $b=c=0.6, \tau_{\sigma}=\tau_{\omega}=$ $0.01 \mu \mathrm{sec}, \bar{v}=0.01 \mathrm{~cm} / \mu \mathrm{sec}$ at $x=0(-), x=L / 2(--)$, and $x=L(-\cdot-)$.

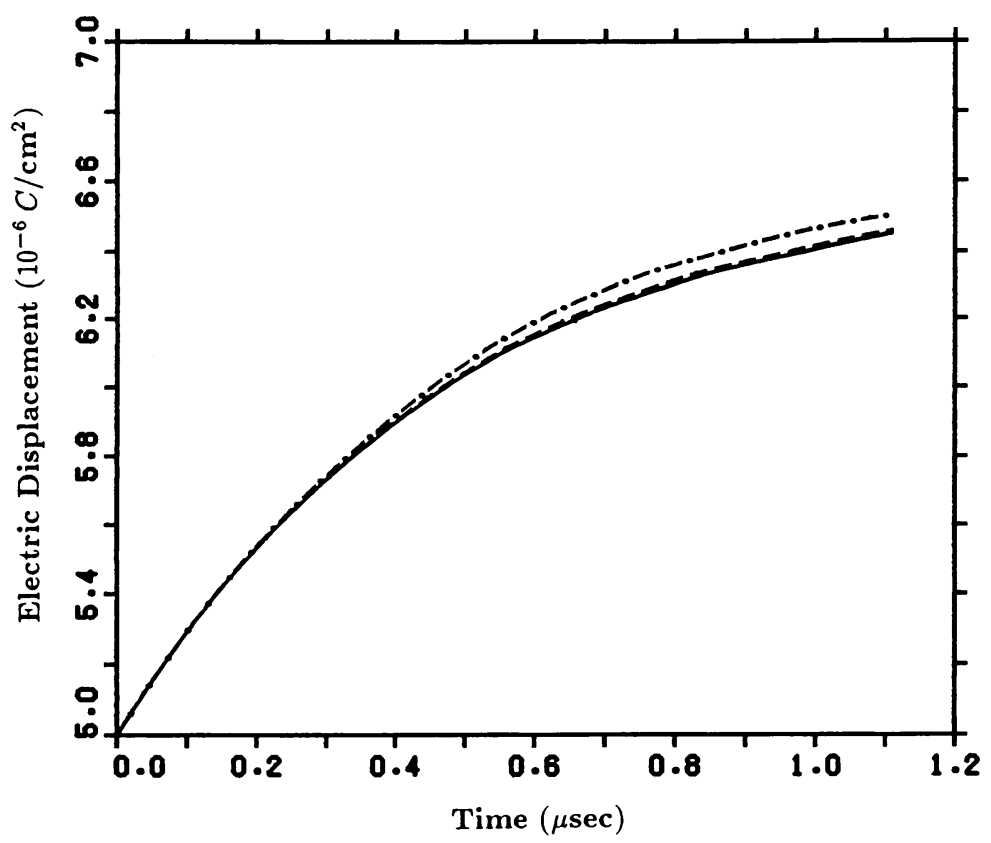

FIG. 4. Electric displacement with time for $b=c=0.4(-), b=$ $c=0.65(--), b=c=1.5(-\cdot-)$, and $\tau_{\sigma}=\tau_{\omega}=0.4 \mu \mathrm{sec}$. 


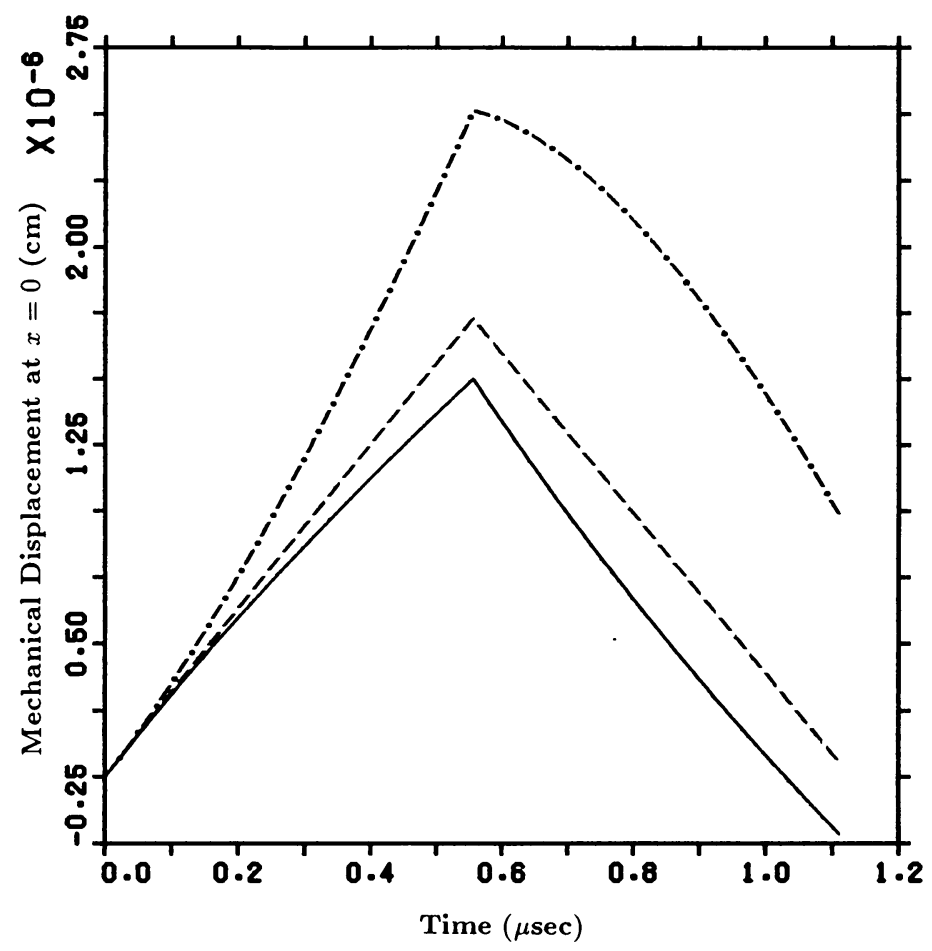

Fig. 5. Mechanical displacement with time at $x=0$ for $b=c=0.4$ $(-), b=c=0.65(--), b=c=1.5(-\cdot-)$, and $\tau_{\sigma}=\tau_{\omega}=$ $0.4 \mu \mathrm{sec}$.

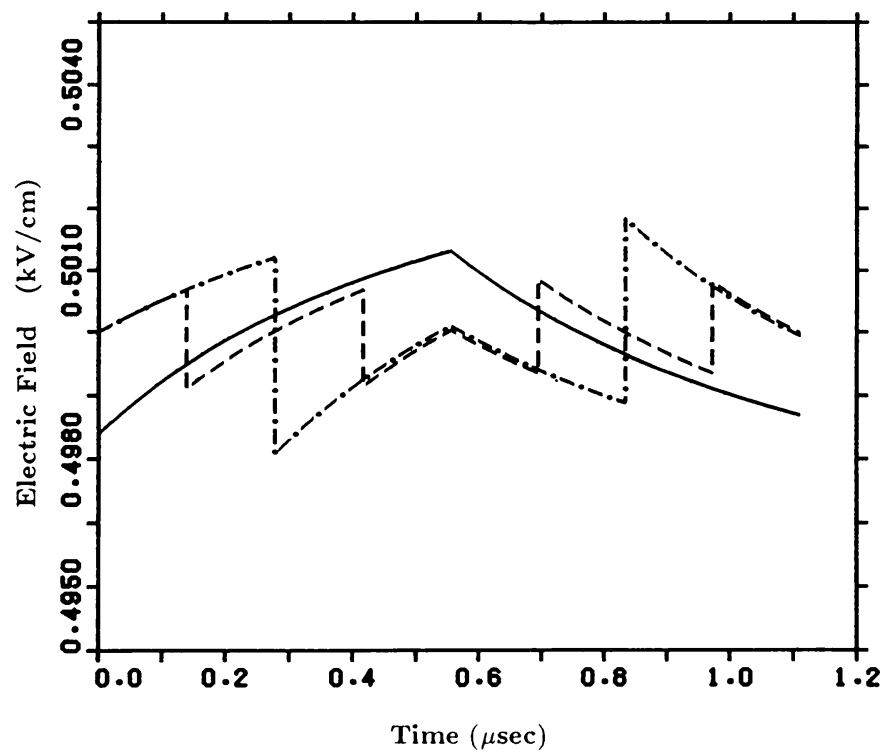

FIG. 6. Electric field with time for $b=c=0.4, \tau_{\sigma}=\tau_{\omega}=0.4 \mu \mathrm{sec}$, at $x=0(-), x=L / 4(-)$, and $x=L / 2 \quad(-\cdot-)$. 


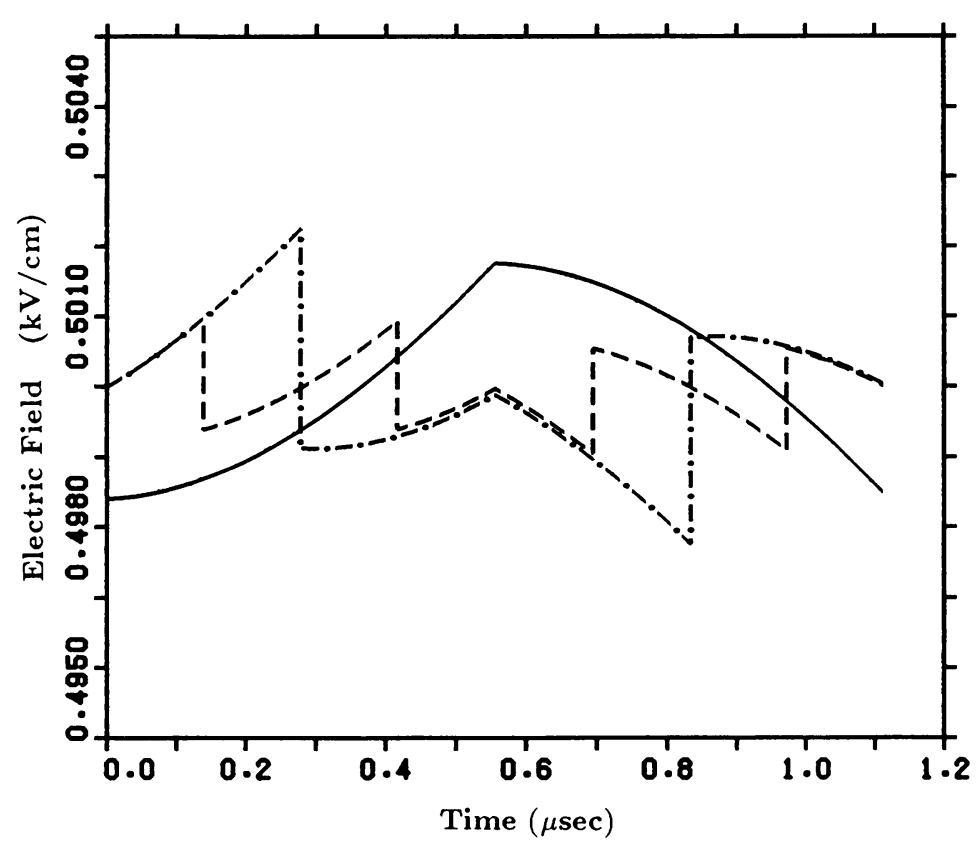

FIG. 7. Electric field with time for $b=c=1.5, \tau_{\sigma}=\tau_{\omega}=1.5 \mu \mathrm{sec}$ at $x=0(-), x=L / 4(-)$, and $x=L / 2(-\cdot-)$.

7. Conclusion. We have demonstrated that the method of geometrical optics provides an efficient algorithm for obtaining both qualitative and quantitative information pertaining to systems involving coupled electro-mechanical effects. Indeed the approach employed here involving, as it does, the use of explicit recursion formulas is very amenable to encoding and rapid numerical evaluation.

Acknowledgment. Preparation of this manuscript was supported in part by an Operating Research Grant A4039 awarded to the second author by the Natural Sciences and Engineering Research Council of Canada and in part by the Killam Foundation in the form of a scholarship awarded to the third author.

\section{REFERENCES}

[1] P. C. Lysne, Dielectric breakdown of shock-loaded PZT 65/35, J. Appl. Phys. 44, 577-582 (1973)

[2] P. C. Lysne and L. C. Bantel, Electromechanical response of PZT 65/35 subjected to axial shock loading, J. Appl. Phys. 46, 222-229 (1975)

[3] P. J. Chen and M. F. McCarthy, One-dimensional shock waves in elastic dielectrics, Instituto Lombardo di Scienze, Rendiconti 107, 715-727 (1973)

[4] D. E. Amos and P. J. Chen, One dimensional linear dynamic electromechanical responses of ferroelectric ceramics, J. Appl. Mech. 45, 749-754 (1978)

[5] P. J. Chen, M. F. McCarthy, and T. R. O'leary, One-dimensional shock and acceleration waves in deformable dielectric materials with memory, Arch. Rational Mech. Anal. 62, 189-207 (1976)

[6] D. E. Amos and P. J. Chen, Linear electromechanical responses of ferroelectric ceramics with inductive-resistive external load, Internat. J. Solids and Structures 15, 319-323 (1979) 
[7] M. F. McCarthy, T. B. Moodie, and R. P. Sawatzky, On the propagation of transients through thermoviscoelastic media, Quart. Appl. Math. 46, 539-557 (1988)

[8] T. S. Öncü and T. B. Moodie, Boundary-initiated wave phenomena in thermoelastic materials, Quart. Appl. Math. 48, 295-320 (1990)

[9] H. J. Sutherland and P. J. Chen, Determination of the mechanical properties of PZT 65/36 using acoustic technique, Acta Mech. 30, 293-298 (1978) 